Pacific Journal of Mathematic 


\title{
ONE-ONE-MAPPINGS ONTO LOCALLY CONNECTED GENERALIZED CONTINUA
}

\author{
Dix H. Pettey
}

\begin{abstract}
This paper is primarily concerned with $1-1$ onto mappings between locally connected generalized continua, especially mappings for which the image spaces are unicoherent. Conditions are given which, if imposed on a space $Y$ guarantee the existence of a nontopological $1-1$ mapping of a locally connected generalized continuum onto $Y$. Other conditions, which prohibit the existence of such a mapping, are also given.
\end{abstract}

Preliminary concepts. By a mapping we will mean a continuous function.

By a generalized continuum we mean a connected, locally connected, locally compact metric space. (It follows from [8, Cor. p. 111] that such a space is always separable.) We shall use LCGC to denote both the expression locally connected generalized continuum and the plural locally connected generalized continua. It follows from a theorem of V. V. Proizvolov [7, Th. 1, p. 1321] that a locally connected, locally compact topological space is metrizable if it has a $1-1$ continuous image in a metric space. Thus, in showing that a space $X$ is a LCGC it is sufficient to show that $X$ is connected, locally connected, and locally compact and that there is a 1-1 mapping of $X$ into a metric space.

For each positive integer $n$, we let $E^{n}, S^{n}$, and $I^{n}$ denote, respectively, Euclidean $n$-space, the unit $n$-sphere, and the unit $n$-cell. The nonnegative real numbers will be denoted as $E_{+}^{1}$, and for $n \geqq 2, E_{+}^{n}$ will denote the topological product of $E^{n-1}$ and $E_{+}^{1}$.

Given a set $H$ in a topological space $X$, we shall let bd $H$ denote the set $\bar{H} \cap(\overline{X-H})$. For an $n$-manifold with boundary $M^{n}$, we shall use $\mathrm{Bd} M^{n}$ to designate the set of all points at which $M^{n}$ is not locally $E^{n}$ (and Int $M^{n}$ to denote $M^{n}-\mathrm{Bd} M^{n}$ ).

Results concerning 1-1 mappings. It is not difficult to find examples of nontopological 1-1 mappings between locally connected generalized continua, especially if we allow the image spaces to be multicoherent. (See, for example, [1, p. 136].) In fact, we have the following theorem.

THEOREm 0. If $Y$ is a multicoherent LCGC then there is a $L C G C$ which can be mapped onto $Y$ by a nontopological $1-1$ mapping.

Proof. Let $H$ and $K$ be closed connected sets in $Y$ with $H \cup K=$ 
$Y$ and $H \cap K$ disconnected. Let $F$ and $F^{\prime \prime}$ be disjoint nonempty closed sets such that $F \cup F^{\prime}=H \cap K$. We shall first show the existence of a closed, locally connected set $L$ such that $F^{\prime} \subset L, F \cap L=\varnothing$, and $L \cup K$ is connected. Since $Y$ is locally compact and paracompact, there is a locally finite closed covering $\mathscr{C}$ of $F^{\prime}$ such that (1) no member of $\mathscr{C}$ intersects $F$, and (2) each member of $\mathscr{C}$ is compact. Now, using $[9,15.43$, p. 22$]$ and $[9,15.3$, p. 20], we can obtain a locally finite collection $\mathscr{L}$ of closed sets such that (1) $\mathscr{L}$ covers the union of the members of $\mathscr{C},(2)$ no member of $\mathscr{L}$ intersects $F$, and (3) each member of $\mathscr{L}$ is locally connected. Let $L^{*}$ denote the union of the members of $\mathscr{L}$. Then $L^{*}$ is closed and locally connected. Now letting $L$ denote the union of the components of $L^{*}$ which intersect $K$, we have $L$ closed and locally connected, $F^{\prime \prime} \subset L, F \cap L=\varnothing$, and $L \cup K$ connected. Since $H$ is connected, there exists a component $V$ of $H-$ $(L \cup K)$ such that $\bar{V}$ intersects both $L$ and $F$. Then $Y-V$ is connected and $Y-(V \cup F)$ is locally connected. If we let $X$ denote the topological space having as points the points of $Y$ and having the topology generated by the open sets of $Y$ and the set $Y-(V \cup F)$, then $X$ is connected, locally connected, and locally compact. Now let $f$ be the identity function on the set $X$. Then $f$ is a nontopological $1-1$ mapping of the space $X$ onto the space $Y$.

If we restrict our consideration to $1-1$ mappings of LCGC onto unicoherent LCGC, it becomes more difficult to find examples in which the mappings are nontopological. In [10], G. T. Whyburn showed that if a $1-1$ mapping of a generalized continuum onto a unicoherent LCGC satisfies one additional relatively mild condition, then the mapping must be a homeomorphism. To more easily describe this condition, we introduce the following notation. For a 1-1 mapping $f$ on a topological space $X$ let $T(f)$ denote the set

$$
\left\{x \in X \mid f^{-1} \text { is not continuous at } f(x)\right\} \text {. }
$$

Whyburn [10, Th. 5, p. 1429] proved that for a 1-1 mapping $f$ of a generalized continuum onto a unicoherent LCGC, if $f \mid T(f)$ is a homeomorphism then $f$ is a homeomorphism. (Observe that for the nontopological mapping $f$ obtained in the proof of Theorem 1, T(f) is a subset of $L$ and the restriction of $f$ to $T(f)$ is a homeomorphism. Thus, in light of Whyburn's theorem, we may conclude that a LCGC $Y$ is multicoherent if and only if there is a LCGC $X$ and a nontopological 1-1 mapping $f$ of $X$ onto $Y$ such that $f \mid T(f)$ is a homeomorphism.)

In [7], V. V. Proizvolov claimed to have proved that if $X$ is a connected, locally connected, locally compact topological space and $Y$ is a unicoherent LCGC then every $1-1$ mapping of $X$ onto $Y$ is a homeomorphism. (See [7, Th. 2, p. 1322].) Examples given by L. C. 
Glaser [2, 3 and 4] and Kenneth Whyburn [11], however, show that this is not the case and that both $E^{3}$ and $S^{3}$ are $1-1$ continuous images of connected 3-manifolds with nonempty boundaries. Making use of one of Glaser's examples, we obtain the following rather general result.

THEOREM 1. If $Y$ is a LCGC and if for some integer $n \geqq 3 Y$ contains an open set $V$ homeomorphic to $E^{n}$, then there is a LCGC $X$ which can be mapped onto $Y$ by a nontopological 1-1 mapping $f$. Furthermore, $X$ and $f$ can be chosen so that the restriction of $f$ to $X-f^{-1}(V)$ is a homeomorphism.

Proof. We may suppose, without loss of generality, that the closure of $V$ in $Y$ is a closed $n$-cell. We shall first obtain a nontopological $1-1$ mapping $\hat{f}$ of a connected $n$-manifold with boundary onto $\bar{V}$ such that $\hat{f} \mid \hat{f}^{-1}(\mathrm{Bd} \bar{V})$ is a homeomorphism. By [3, Lemma 1 , p. 180] there exists a $1-1$ mapping $h$ of $E_{+}^{3}$ onto $S^{3}$. Choose a closed 3-cell $K^{3}$ in Int $E_{+}^{3}$ such that $S^{3}-\operatorname{Int} h\left(K^{3}\right)$ is a closed 3-cell, and let $M^{3}=E_{+}^{3}$ - Int $K^{3}$ and $g=h \mid M^{3}$. Then $g$ is a nontopological 1-1 mapping of the connected 3-manifold with boundary $M^{3}$ onto the closed 3-cell $S^{3}-\operatorname{Int} h\left(K^{3}\right)$. If $n=3$ then, since $g^{-1}\left(\mathrm{Bd} g\left(M^{3}\right)\right)=$ Bd $K^{3}$ and since $g \mid \mathrm{Bd} K^{3}$ is certainly a homeomorphism, we can obtain the desired mapping $\hat{f}$ simply by choosing a homeomorphism $\phi$ of $g\left(M^{3}\right)$ onto $\bar{V}$ and letting $\hat{f}=\phi g$. If $n>3$ we proceed as follows. Since $g\left(M^{3}\right)$ is a closed 3-cell we may regard $I^{n}$ to be $g\left(M^{3}\right) \times I^{n-3}$. Define $\lambda: M^{3} \times I^{n-3} \rightarrow I^{n}$ by letting $\lambda(r, s)=(g(r), s)$ for each $r \in M^{3}, s \in I^{n-3}$. Let $J^{n}$ be the space obtained from $I^{n} \times\{1,2\}$ by identifying the point $(q, 1)$ with the point $(q, 2)$ for each $q \in \mathrm{Bd} I^{n}$. Let $M_{0}^{n}$ be the space obtained from $\left(M^{3} \times I^{n-3}\right) \times\{1,2\}$ by identifying the point $(p, 1)$ with the point $(p, 2)$ for each $p \in \lambda^{-1}\left(\mathrm{Bd} I^{n}\right)$. Then $M_{0}^{n}$ is a connected $n$ manifold with boundary and $J^{n}$ is an $n$-sphere. Define $\mu: M_{0}^{n} \rightarrow J^{n}$ by letting $\mu(p, i)=(\lambda(p), i)$ for each $p \in M^{3} \times I^{n-3}, i \in\{1,2\}$. Now choose a closed $n$-cell $K^{n}$ in Int $M_{0}^{n}$ such that $J^{n}-\operatorname{Int} \mu\left(K^{n}\right)$ is a closed $n$ cell, and let $M^{n}=M_{0}^{n}$ - Int $K^{n}$ and $\sigma=\mu \mid M^{n}$. Then $\sigma$ is a nontopological $1-1$ mapping of the connected $n$-manifold with boundary $M^{n}$ onto the closed $n$-cell $J^{n}-$ Int $\mu\left(K^{n}\right)$, and the restriction of $\sigma$ to $\sigma^{-1}\left(\mathrm{Bd} \sigma\left(M^{n}\right)\right)\left(=\mathrm{Bd} K^{n}\right)$ is a homeomorphism. The desired $\hat{f}$ is now obtained by letting $\theta$ be a homeomorphism of $\sigma\left(M^{n}\right)$ onto $\bar{V}$ and then letting $\hat{f}=\theta \sigma$. To complete our proof of the theorem we let $X$ be the space obtained from the free union of $M^{n}$ and $Y-V$ by identifying the point $t$ with the point $\hat{f}^{-1}(t)$ for each $t \in \mathrm{Bd} \bar{V}$. Then $X$ is connected, locally connected, and locally compact. If we define $f: X \rightarrow Y$ by letting $f \mid Y-V$ be the identity and $f \mid M^{n}=\hat{f}$, then $f$ is a nontopological $1-1$ mapping of $X$ onto $Y$ and the restriction of $f$ to $X-f^{-1}(V)$ is a homeomorphism. 
A consideration of $1-1$ mappings onto 1-dimensional spaces yields a result of a quite different nature. In contrast to Theorem 1, we have the following.

THEOREM 2. If $X$ is a connected, peripherally compact topological space and $Y$ is a 1-dimensional unicoherent LCGC, then every $1-1$ mapping of $X$ onto $Y$ is a homeomorphism. (A topological space is said to be peripherally compact if there is a base $\mathscr{C}$ for the space with bd $U$ compact for each $U \in \mathscr{Q}$.)

Proof. Due to a result of G. T. Whyburn [10, Cor. p. 1428], we need only show that $Y$ contains no simple closed curve. Assume the contrary and let $J$ be such a simple closed curve. Then there is a connected open set $V$ in $Y$ such that bd $V$ is 0 -dimensional and such that each of $V \cap J$ and $J-\bar{V}$ is nonempty. Let $G$ denote a component of $J-\bar{V}$ and let $W$ denote the component of $Y-\bar{V}$ which contains $G$. Since bd $G$ consists of two points and since $\bar{G} \cap \bar{V} \subset$ bd $W \subset$ bd $V$, we conclude that $\bar{G} \cap \bar{V}(=\bar{W} \cap(Y-W))$ is disconnected. But the connectedness of $\bar{V}$ implies that $Y-W$ is connected, so $Y$ is the union of two closed connected sets, $\bar{W}$ and $Y-W$, having a disconnected intersection. This contradicts the hypothesis that $Y$ is unicoherent.

For 1-1 mappings onto 2-dimensional LCGC the situation is somewhat more complicated. Theorem 1 cannot be extended to include the case $n=2$ since none of the three most obvious examples of 2dimensional LCGC (i.e., $E^{2}, S^{2}$, and $I^{2}$ ) is the nontopological $1-1$ continuous image of a LCGC. (See [5, Th. 4.3, p. 305] and [6, Cor. 1.1 and 1.2, p. 277].) More generally, we have the following.

THEOREM 3. Let $X$ be a LCGC and let $Y$ be a LCGC having the following property: for each simple closed curve $J$ in $Y$ there is a closed 2-cell $K$ in $Y$ such that $J=\mathrm{Bd} K$ and such that Int $K$ is an open set in $Y$. Then every $1-1$ mapping of $X$ onto $Y$ is a homeomorphism. (For a proof, see [6, Th. 1, p. 277].)

Since a unicoherent LCGC in $E^{2}$ (or $S^{2}$ ) will always have the property required in the hypothesis of Theorem 3, we have the following corollary. (Compare [6, Cor. 1.3, p. 277].)

CoRollary 3.1. If $X$ is a LCGC and $Y$ is a unicoherent LCGC in $E^{2}\left(\right.$ or $\left.S^{2}\right)$ then every $1-1$ mapping of $X$ onto $Y$ is a homeomorphism.

There are, however, examples of nontopological $1-1$ mappings of LCGC onto 2-dimensional unicoherent LCGC. One such example is 
given by Duda [1, p. 137]. Another is the following.

ExAmple A. Let $I_{0}$ be the half-open interval $(-1,0]$ on the $y$ axis in $E^{3}$, and for each positive integer $n$, let $I_{n}$ denote the interval $(n-1, n]$ on the $y$-axis, $I_{n}^{\prime}$ the interval $(0,1)$ on the line $x=0, y=$ $n$, and $V_{n}$ the open 2-cell $x^{2}+(y-n)^{2}<1 / 9$ in the plane $z=1$. (See Figure 1(a).) Let $X=\left(\bigcup_{n=0}^{\infty} I_{n}\right) \cup\left(\bigcup_{n=1}^{\infty} I_{n}^{\prime}\right) \cup\left(\bigcup_{n=1}^{\infty} V_{n}\right)$. Then $X$ is a LCGC. Now let $Y$ be the union of closed 2-cells $K_{1}, K_{2}, K_{3}, \cdots$ such that for each $n$ (1) $K_{n}$ does not intersect the closure of $\bigcup_{i=n+2}^{\infty} K_{i}$, (2) $K_{n} \cap K_{n+1}$ is an arc in $\mathrm{Bd} K_{n+1}$, and (3) $K_{n} \cap K_{n+1}$ intersects $\mathrm{Bd} K_{n}$ at exactly one point, this being an end-point of $K_{n} \cap K_{n+1}$. (See Figure 1(b).) Then $Y$ is a 2-dimensional, unicoherent LCGC. The space $X$ can be taken onto $Y$ by a $1-1$ mapping $f$ such that $f\left(I_{0}\right)=\mathrm{Bd} K_{1}$ (with $\left.f(0,0,0)=\mathrm{Bd} K_{1} \cap \mathrm{Bd} K_{2}\right), f\left(V_{1}\right)=\operatorname{Int} K_{1}, f\left(I_{1} \cup I_{1}^{\prime}\right)=\left(\operatorname{Bd} K_{2}\right)-$ $K_{1}, \cdots, f\left(V_{n}\right)=\operatorname{Int} K_{n}, f\left(I_{n} \cup I_{n}^{\prime}\right)=\left(\operatorname{Bd} K_{n+1}\right)-K_{n}, \cdots$ (Figure 1).

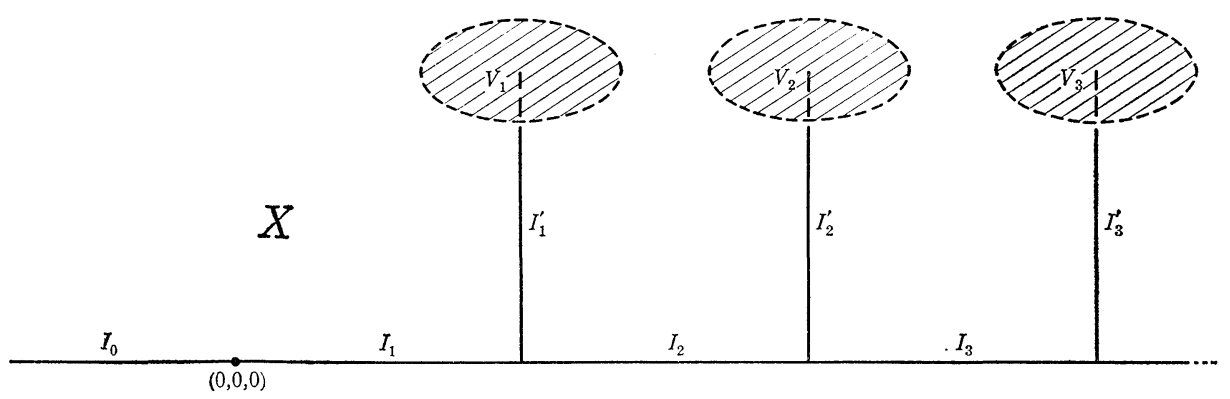

(a)

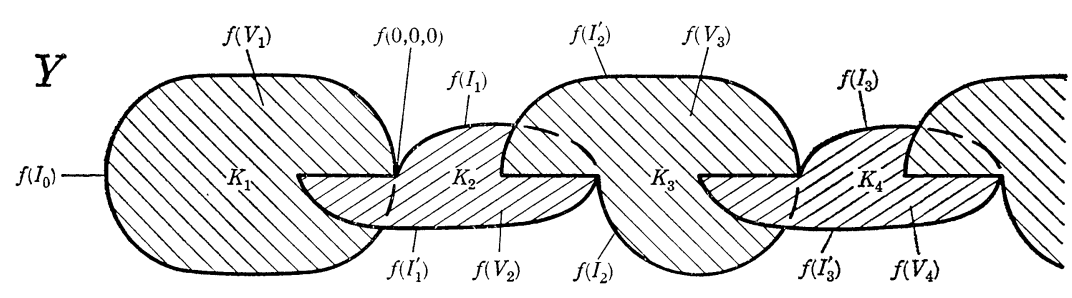

(b)

Figure 1

(We remark here, without going into detail, that a slight modification of Example A produces another interesting mapping. If we thicken $X$ and $Y$ so that $Y$ becomes a topological copy of $E^{3}$ and $X$ a 3-manifold with boundary - with only enough boundary so that we can still map the thickened $X$ onto the thickened $Y$ in a $1-1$ continuous manner - then the thickened $X$ will be homeomorphic to $E_{+}^{3}$ and the 1-1 mapping onto $E^{3}$ will be equivalent to that described - in quite a different manner - by Glaser in [3].) 
In seeking to identify significant properties of the space $Y$ in Example A - properties which allow for the existence of a nontopological 1 - 1 mapping of a LCGC onto $Y$ - our attention is drawn to the special way in which each 2-cell $K_{n}$ intersects its successor $K_{n+1}$. Indeed, if $Y$ were modified so that each of these intersections were an arc in $\mathrm{Bd} K_{n} \cap \mathrm{Bd} K_{n+1}$, then $Y$ would be a topological copy of $E_{+}^{2}$ and by Corollary 3.1 no such mapping would be possible. Nor could such a mapping exist if each $K_{n} \cap K_{n+1}$ were a single point. In order to prove this last statement (Corollary 4.1 below) we first state and prove the following theorem, which is of some interest for its own sake in the study of $1-1$ mappings.

TheOREM 4. Suppose that $Y$ is a LCGC and that $Y=\bigcup_{\beta \in S} Y_{\beta}$, where for each $\beta \in \mathscr{B}$ (1) $Y_{\beta}$ is a closed subset of $Y$, (2) $Y_{\beta} \cap \overline{Y-Y_{\beta}}$ is a point, and (3) $Y_{\beta}$ is not the image, under a nontopological 1-1 mapping, of a LCGC. Then $Y$ is not the image, under a nontopological 1 - 1 mapping, of a LCGC.

Proof. Assume the contrary and let $f$ be a nontopological $1-1$ mapping of a LCGC $X$ onto $Y$. By [9, 5.2, p. 38] $X$ is arcwise connected. Therefore, by [10, Th. 7, p. 1430], there is a topological ray $\alpha$ in $X$ such that $f(\alpha)$ is a simple closed curve. From (2) in the hypothesis, it follows that for some $\gamma \in \mathscr{B}, f(\alpha) \subset Y_{r}$. Let $q=$ $Y_{r} \cap{\overline{Y-Y_{\gamma}}}_{\text {. Then }} f^{-1}(q)$ separates $f^{-1}\left(Y_{r}-q\right)$ from $X-f^{-1}\left(Y_{r}\right)$, which implies that $f^{-1}\left(Y_{r}\right)$ is connected. By a similar argument, if $V$ is a connected open set in $X$, then $V \cap f^{-1}\left(Y_{\gamma}\right)$ is connected. So $f^{-1}\left(Y_{\gamma}\right)$ is locally connected. And since $f^{-1}\left(Y_{\gamma}\right)$ is closed in $X, f^{-1}\left(Y_{\gamma}\right)$ is locally compact. It now follows from (3) in the hypothesis that the restriction of $f$ to $f^{-1}\left(Y_{\gamma}\right)$ is a homeomorphism. But since $\alpha \subset f^{-1}\left(Y_{\gamma}\right)$ and since $f \mid \alpha$ is not a homeomorphism this gives us a contradiction.

CoRollary 4.1. If $X$ is a LCGC and if $Y$ is the union of 2-cells $K_{1}, K_{2}, K_{3}, \cdots$ such that for each $n(1) K_{n}$ does not intersect the closure of $K_{n+2} \cup K_{n+3} \cup \cdots$, and (2) $K_{n} \cap K_{n+1}$ is a point, then every $1-1$ mapping of $X$ onto $Y$ is a homeomorphism.

Proof. For each $n$, let $Y_{n}=K_{1} \cup \cdots \cup K_{n}$. Since no $K_{n}$ is the image, under a nontopological $1-1$ mapping, of a LCGC (Corollary 3.1 or [6, Cor. 3.2, p. 277]), it follows from Theorem 4, by induction, that no $Y_{n}$ is the image, under a nontopological 1-1 mapping, of a LCGC. Since $Y=\bigcup_{n=1}^{\infty} Y_{n}$, a second application of Theorem 4 now tells us that $Y$ is not the image, under a nontopological 1-1 mapping, of a LCGC. 
In Example $\mathrm{A}$ the $K_{n}$ 's intersect in such a way that each union $K_{n} \cup K_{n+1}$ contains a set which is topologically the product of an arc and a simple triod. We shall call such a set a book-with-3-pages. (The image space in Duda's example [1, p. 137] also contains a book-with3-pages.)

A book-with-3-pages itself is the simplest example known to the author of a 2-dimensional unicoherent LCGC which is the nontopological $1-1$ continuous image of a LCGC. We shall now describe a nontopological 1-1 mapping of a LCGC onto a book-with-3-pages and then refer to this mapping in proving a more general theorem (Theorem 5 , below) concerning spaces which contain books-with-3-pages.

Example B. In the plane $z=2$ in $E^{3}$, let $K$ denote the closed

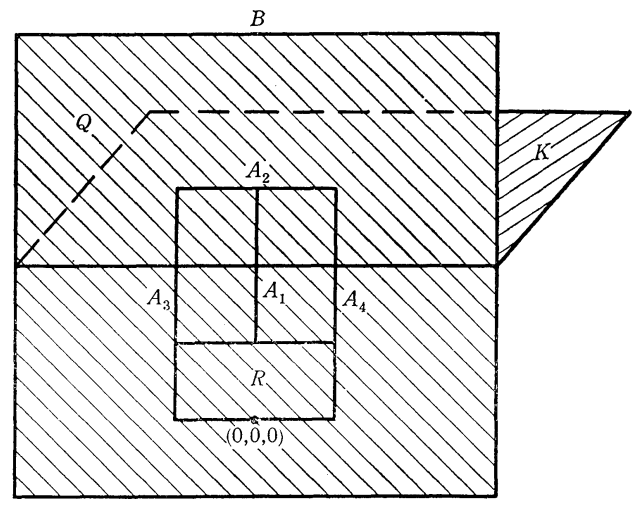

(a)

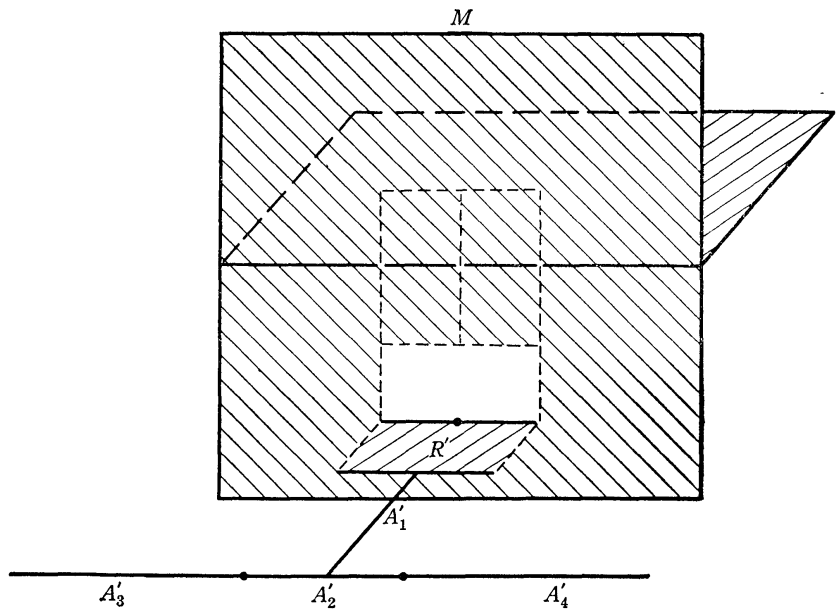

(b)

FIGURE 2 
rectangular set $\{(x, y, 2) \mid-3 \leqq x \leqq 0,-3 \leqq y \leqq 3\}$. In the plane $x=0$, let $Q$ denote the closed square set $\{(0, y, z) \mid-3 \leqq y \leqq 3,-1 \leqq z \leqq 5\}$. Let $B=K \cup Q$ (Figure 2(a)). Then $B$ is a book-with-3-pages.

In the plane $x=0$, define sets $R, A_{1}, A_{2}, A_{3}$, and $A_{4}$ as follows (see Figure 2(a)):

( 0 ) $R$ is the rectangular set $\{(0, y, z) \mid-1<y<1,0<z \leqq 1\}$;

(1) $A_{1}$ is the closed straight-line segment from $(0,0,1)$ to $(0,0,3)$;

(2) $A_{2}$ is the closed straight-line segment from $(0,-1,3)$ to $(0,1,3)$;

(3) $A_{3}$ is the closed straight-line segment from $(0,-1,3)$ to $(0,-1,0)$;

(4) $A_{4}$ is the closed straight-line segment from $(0,1,3)$ to $(0,1,0)$. Let $\widetilde{B}=B-\left(R \cup A_{1} \cup A_{2} \cup A_{3} \cup A_{4}\right)$. Now in the plane $z=0$, define sets $R^{\prime}, A_{1}^{\prime}, A_{2}^{\prime}, A_{3}^{\prime}$, and $A_{4}^{\prime}$ as follows (see Figure 2(b)):

( 0 ) $R^{\prime}$ is the rectangular set $\{(x, y, 0) \mid 0<x \leqq 1,-1<y<1\}$;

(1) $A_{1}^{\prime}$ is the closed straight-line segment from $(1,0,0)$ to $(3,0,0)$;

(2) $A_{2}^{\prime}$ is the closed straight-line segment from $(3,-1,0)$ to $(3,1,0)$;

(3) $A_{3}^{\prime}$ is the closed straight-line segment from $(3,-1,0)$ to $(3,-4,0)$;

(4) $A_{4}^{\prime}$ is the closed straight-line segment from $(3,1,0)$ to $(3,4,0)$. Let $M=\widetilde{B} \cup R^{\prime} \cup A_{1}^{\prime} \cup A_{2}^{\prime} \cup A_{3}^{\prime} \cup A_{4}^{\prime}$. Then $M$ is a LCGC topologically different from $B$.

We can now define a function $m$ from $M$ to $B$ such that

(1) the restriction of $m$ to $\widetilde{B}$ is the identity mapping,

(2) on $R^{\prime} \cup A_{1}^{\prime} \cup A_{2}^{\prime}, m$ is given by $m(x, y, 0)=(0, y, x)$,

(3) on $A_{3}^{\prime}, m$ is given by $m(3, y, 0)=(0,-1,4+y)$, and

(4) on $A_{4}^{\prime}, m$ is given by $m(3, y, 0)=(0,1,4-y)$.

Then $m$ is a $1-1$ mapping which takes $\widetilde{B}$ onto itself, $R^{\prime}$ onto $R, A_{1}^{\prime}$ onto $A_{1}, A_{2}^{\prime}$ onto $A_{2}, A_{3}^{\prime}$ onto $A_{3}$, and $A_{4}^{\prime}$ onto $A_{4}$.

In the proof of the following theorem $B, R, A_{1}, A_{2}, A_{3}, A_{4}$, and $M$ will denote the same sets, and $m$ the same mapping, as in Example B.

THeorem 5. If $Y$ is a LCGC which contains a book-with-3-pages $B^{\prime}$ and if there is an open set $V$ in $Y$ such that $V \subset B^{\prime}$, then there is a LCGC $X$ which can be mapped onto $Y$ by a nontopological 1-1 mapping $f$. Furthermore, $X$ and $f$ can be chosen so that the restriction of $f$ to $X-f^{-1}\left(B^{\prime}\right)$ is a homeomorphism.

Proof. We may assume, without loss of generality, that $V$ is an open 2-cell. Let $\alpha$ denote the union of ares $A_{2}, A_{3}$, and $A_{4}$ in the book-with-3-pages $B$. There exists an uncountable collection $\mathscr{H}$ of homeomorphisms of $B$ onto $B^{\prime}$ such that (1) each member of $\mathscr{C}$ takes $\bar{R}$ into $V$, and (2) if $h_{1}, h_{2} \in \mathscr{C}$ and $h_{1} \neq h_{2}$ then $h_{1}(\alpha) \cap h_{2}(\alpha)=\varnothing$. 
Since $\alpha$ does not separate $B$, it follows from [9, Th. 1.5, p. 43] that for some $\hat{h} \in \mathscr{H}, \hat{h}(\alpha)$ does not separate $Y$. Now let $\mathscr{G}$ be an uncountable collection of homeomorphisms of $B$ onto $B^{\prime}$ such that (1) each member of $\mathscr{G}$ takes $\alpha \cup R$ onto $\hat{h}(\alpha \cup R)$, and (2) if $g_{1}, g_{2} \in \mathscr{G}$ and $g_{1} \neq g_{2}$ then $g_{1}\left(A_{1}\right) \cap g_{2}\left(A_{1}\right)=\varnothing$. Since $A_{1}-\alpha$ does not separate $B-\alpha$, it follows that for some $\hat{g} \in \mathscr{G}, \hat{g}\left(A_{1}-\alpha\right)$ does not separate $Y-\hat{h}(\alpha)$, i.e., $\hat{g}\left(\alpha \cup A_{1}\right)$ does not separate $Y$. Since $\hat{g}(\bar{R}) \subset V$, we may conclude that $\hat{g}\left(\alpha \cup A_{1} \cup R\right)$ does not separate $Y$. Now $B-\left(\alpha \cup A_{1} \cup R\right)$ is the intersection of $B$ and the LCGC $M$ (from Example B). Let $X$ denote the topological space obtained from the free union of $M$ and

$$
Y-\widehat{g}\left(\alpha \cup A_{1} \cup R\right)
$$

by identifying the point $p$ with the point $\hat{g}(p)$ for each $p \in M \cap B$. Then $X$ is connected, locally connected, and locally compact. Finally (with $m$ still denoting the above $1-1$ mapping of $M$ onto $B$ ), we define $f: X \rightarrow Y$ by letting

$$
f(q)=\left\{\begin{array}{l}
\hat{g} m(q) \text { if } q \in M \\
q \quad \text { if } q \notin M .
\end{array}\right.
$$

Then $f$ is a nontopological $1-1$ mapping of $X$ onto $Y$, and the restriction of $f$ to $f^{-1}\left(Y-B^{\prime}\right)$ is a homeomorphism.

CoROLLARY 5.1. If a LCGC $Y$ is the union of countably many books-with-3-pages, then there is a LCGC which can be mapped onto $Y$ by a nontopological $1-1$ mapping.

Proof. By Baire's theorem, one of the countably many bookswith-3-pages contains a set which is open in $Y$. Thus, the desired conclusion follows from Theorem 5 .

As special cases of both Theorem 5 and Corollary 5.1 we have the following results, which provide us with some additional examples of rather simple 2-dimensional unicoherent LCGC which are nontopological $1-1$ continuous images of LCGC.

COROLlary 5.2. Let $n$ be an integer greater than 2. If a metric space $Y$ is the union of $n$ 2-cells $K_{1}, K_{2}, \cdots, K_{n}$ such that $K_{i} \cap K_{j}=$ $\mathrm{Bd} K_{i}=\mathrm{Bd} K_{j}$ whenever $1 \leqq i<j \leqq n$, then there is a LCGC which can be mapped onto $Y$ by a nontopological $1-1$ mapping.

CoROLlary 5.3. Let $n$ be an integer greater than 2. If a metric space $Y$ is the union of $n$ 2-cells $K_{1}, K_{2}, \cdots, K_{n}$ such that for some arc $A$ in $Y, K_{i} \cap K_{j}=\mathrm{Bd} K_{i} \cap \mathrm{Bd} K_{j}=A$ whenever $1 \leqq i<j \leqq n$, then there 
is a LCGC which can be mapped onto $Y$ by a nontopological $1-1$ mapping. (Note that for the special case $n=3, Y$ is just a bookwith-3-pages.)

QUESTION. Can the requirement that $B^{\prime}$ contain an open set be deleted from the hypothesis of Theorem 5 ?

QUESTION. If $Y$ is a unicoherent LCGC which does not contain a book-with-3-pages, then must every $1-1$ mapping of a LCGC onto $Y$ be a homeomorphism?

\section{REFERENCES}

1. Edwin Duda, One-to-one mappings and applications, General Topology and its Applications, 1 (1971), 135-142.

2. L. C. Glaser, $1-1$ continuous mappings onto $E^{n}$, Amer. J. Math., 88 (1966), 237-243.

3. Dimension lowering monotone noncompact mappings of $E^{n}$, Fund. Math., 58 (1966), 177-181.

4. - Bing's house with two rooms from a 1-1 continuous map onto $E^{3}$, Amer. Math. Monthly, 74 (1967), 156-160.

5. Dix H. Pettey, Mappings onto the plane, Trans, Amer. Math. Soc., 157 (1971), 297309.

6. - One-io-one mappings, Proc. Amer. Math. Soc., 31 (1972), 276-278.

7. V. V. Priozvolov, One-to-one mappings onto metric spaces, Soviet Math. Dokl., 5 (1964), 1321-1322, Amer. Math. Soc. translation from Dokl. Akad. Nauk. SSSR, 158 (1964), 788-789.

8. W. Sierpeński, Sur les espaces métriques localement séparables, Fund. Math., 21 (1933), 107-113.

9. G. T. Whyburn, Analytic Topology, 2nd ed., Amer. Math. Soc. Colloq. Publ., vol. 38, Amer. Math. Soc., Providence, R. I., 1963.

10. - On compactness of mappings, Proc. Nat. Acad. Sci. U.S.A., 52 (1964), $1426-1431$.

11. Kenneth Whyburn, A nontopological 1-1 mapping onto $E^{3}$, Bull. Amer. Math. Soc., 71 (1965), 533-537.

Received October 16, 1972 and in revised form August 4, 1973.

UNIVERSITY OF MISSOURI 


\section{PACIFIC JOURNAL OF MATHEMATICS}

\section{EDITORS}

RICHARD ARENS (Managing Editor)

University of California

Los Angeles, California 90024

\section{R. A. Beaumont \\ University of Washington \\ Seattle, Washington 98105}

\section{J. DugundjI*}

Department of Mathematics University of Southern California Los Angeles, California 90007

D. Gilbarg and J. Milgram

Stanford University

Stanford, California 94305

\section{ASSOCIATE EDITORS}
E. F. BeCKenBaCH
B. H. NeumanN
F. WOLF
K. YoSHIDA

\section{SUPPORTING INSTITUTIONS}

\author{
UNIVERSITY OF BRITISH COLUMBIA \\ CALIFORNIA INSTITUTE OF TECHNOLOGY \\ UNIVERSITY OF CALIFORNIA \\ MONTANA STATE UNIVERSITY \\ UNIVERSITY OF NEVADA \\ NEW MEXICO STATE UNIVERSITY \\ OREGON STATE UNIVERSITY \\ UNIVERSITY OF OREGON \\ OSAKA UNIVERSITY
}

\author{
UNIVERSITY OF SOUTHERN CALIFORNIA \\ STANFORD UNIVERSITY \\ UNIVERSITY OF TOKYO \\ UNIVERSITY OF UTAH \\ WASHINGTON STATE UNIVERSITY \\ UNIVERSITY OF WASHINGTON

$* * *$
$*$
AMERICAN MATHEMATICAL SOCIETY
NAVAL WEAPONS CENTER

The Supporting Institutions listed above contribute to the cost of publication of this Journal, but they are not owners or publishers and have no responsibility for its content or policies.

Mathematical papers intended for publication in the Pacific Journal of Mathematics should be in typed form or offset-reproduced, (not dittoed), double spaced with large margins. Underline Greek letters in red, German in green, and script in blue. The first paragraph or two must be capable of being used separately as a synopsis of the entire paper. Items of the bibliography should not be cited there unless absolutely necessary, in which case they must be identified by author and Journal, rather than by item number. Manuscripts, in duplicate if possible, may be sent to any one of the four editors. Please classify according to the scheme of Math. Rev. Index to Vol. 39. All other communications to the editors should be addressed to the managing editor, or Elaine Barth, University of California, Los Angeles, California, 90024.

100 reprints are provided free for each article, only if page charges have been substantially paid. Additional copies may be obtained at cost in multiples of 50 .

The Pacific Journal of Mathematics is issued monthly as of January 1966. Regular subscription rate: $\$ 60.00$ a year (6 Vols., 12 issues). Special rate: $\$ 30.00$ a year to individual members of supporting institutions.

Subscriptions, orders for back numbers, and changes of address should be sent to Pacific Journal of Mathematics, 103 Highland Boulevard, Berkeley, California, 94708.

PUBLISHED BY PACIFIC JOURNAL OF MATHEMATICS, A NON-PROFIT CORPORATION

Printed at Kokusai Bunken Insatsusha (International Academic Printing Co., Ltd.), 270, 3-chome Totsuka-cho, Shinjuku-ku, Tokyo 160, Japan

* C. R. DePrima California Institute of Technology, Pasadena, CA 91109, will replace J. Dugundji until August 1974. 


\section{Pacific Journal of Mathematics}

Vol. 50, No. $2 \quad$ October, 1974

Mustafa Agah Akcoglu, John Philip Huneke and Hermann Rost, A counter example to the Blum Hanson theorem in general spaces .............

Huzihiro Araki, Some properties of modular conjugation operator of von

Neumann algebras and a non-commutative Radon-Nikodym theorem

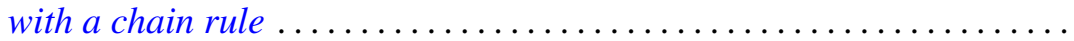

E. F. Beckenbach, Fook H. Eng and Richard Edward Tafel, Global properties of rational and logarithmico-rational minimal surfaces .....

David W. Boyd, A new class of infinite sphere packings ............. 383

K. G. Choo, Whitehead Groups of twisted free associative algebras ........

Charles Kam-Tai Chui and Milton N. Parnes, Limit sets of power series

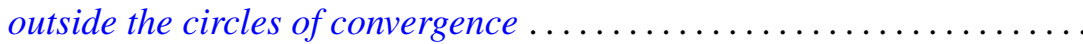

Allan Clark and John Harwood Ewing, The realization of polynomial

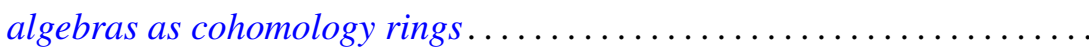

Dennis Garbanati, Classes of circulants over the p-adic and rational

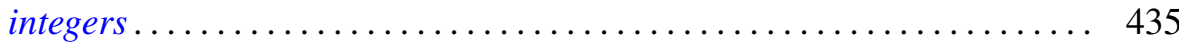

Arjun K. Gupta, On a "square" functional equation ................... 449

David James Hallenbeck and Thomas Harold MacGregor, Subordination and extreme-point theory ............................. 455

Douglas Harris, The local compactness of $v X \ldots \ldots . . . . . . . . . . . .4469$

William Emery Haver, Monotone mappings of a two-disk onto itself which fix the disk's boundary can be canonically approximated by homeomorphisms .................................. 477

Norman Peter Herzberg, On a problem of Hurwitz .................. 485

Chin-Shui Hsu, A class of Abelian groups closed under direct limits and subgroups formation ............................... 495

Bjarni Jónsson and Thomas Paul Whaley, Congruence relations and multiplicity types of algebras.....................

Lowell Duane Loveland, Vertically countable spheres and their wild sets.

Nimrod Megiddo, Kernels of compound games with simple components ....

Russell L. Merris, An identity for matrix functions ........ . .

E. O. Milton, Fourier transforms of odd and even tempered distributions ...

Dix Hayes Pettey, One-one-mappings onto locally connected generalized continua

Mark Bernard Ramras, Orders with finite global dimension

Doron Ravdin, Various types of local homogeneity. .

George Michael Reed, On metrizability of complete Moore spaces ...

Charles Small, Normal bases for quadratic extensions ..

Philip C. Tonne, Polynomials and Hausdorff matrices.... . . 